\title{
WORONOWICZ TANNAKA-KREIN DUALITY AND FREE ORTHOGONAL QUANTUM GROUPS
}

\author{
SARA MALACARNE
}

\begin{abstract}
Given a finite dimensional Hilbert space $H$ and a collection of operators between its tensor powers satisfying certain properties, we give a short proof of the existence of a compact quantum group $G$ with a fundamental representation $U$ on $H$ such that the intertwiners between the tensor powers of $U$ coincide with the given collection of operators. We then explain how the general version of Woronowicz Tannaka-Krein duality can be deduced from this.
\end{abstract}

\section{INTRODUCTION}

The aim of the paper is to give a short proof of Woronowicz Tannaka-Krein duality Theorem [8] using only basic tools of finite dimensional algebra. We consider a finite dimensional Hilbert space $H$ and a collection of operators between its tensor powers satisfying certain properties. Categorically speaking, we deal with a $\mathrm{C}^{*}$-tensor category with conjugates that is a subcategory of the category of finite dimensional Hilbert spaces, Hilbf, and assume that such category is generated by one self-conjugate Hilbert space. We prove the existence of a compact quantum group $G$, such that its representation category $\operatorname{Rep} G$ is our given category. The proof consists of an explicit reconstruction of the Hopf $*$-algebra $\mathrm{C}[G]$, generated by the coefficients of all finite dimensional representations of $G$. The relations defining such Hopf $*-$ algebra are directly obtained through morphisms in the category, or equivalently, through the collection of operators between tensor powers of $H$. The version of Woronowicz Tannaka-Krein Theorem that we prove is essentially formulated in the paper by T. Banica and R. Speicher [2], where the duality is used for the construction of new examples of free quantum groups, via subcategories of the so-called categories of noncrossing partitions. Even though the proof here presented is, in many respects, similar to the proofs of Woronowicz Tannaka-Krein duality appearing in [8] and [4], we wish to point out that this version is more algebraic, mostly category-free and the key part is based on simple duality statements for finite dimensional vector spaces. Related to this reconstruction process, it is also important to mention $\mathrm{P}$. Schauenburg's paper [5], in which a proof of Tannaka-Krein duality is given in a more general setting: monoidal categories that are not semisimple are considered and, correspondingly, arbitrary Hopf algebras are recovered. We refer to [3] for a general introduction to Tannaka-Krein duality and the more general Woronowicz Tannaka-Krein duality theorem.

The paper is structured in the following way: in Section 1 we define a bialgebra, which we will later prove to be the Hopf $*$-algebra $C[G]$. The relations defining this bialgebra are obtained from the collection of operators between tensor powers of $H$, denoted by $\mathcal{C}$. In Section 2 we prove that the bialgebra defined in Section 1 can be equipped with a Hopf $*$-algebra structure. For this we first consider a smaller collection of operators, $\mathcal{C}_{F}$, and show that they define the free orthogonal quantum group $O_{F}^{+}$, [7]. We want to stress that nothing, apart from the fact that $\mathrm{C}\left[O_{F}^{+}\right]$is a well-defined Hopf *-algebra, is used. In Section 3 we prove the equivalence between the $\mathrm{C}^{*}$-tensor category generated by one selfdual Hilbert space $H$ and $\operatorname{Rep} G$. In Section 4 we show how the particular case analysed in Section 3 can be extended to the general case of a not necessarily finitely generated $\mathrm{C}^{*}$-tensor category.

Acknowledgements. I would like to thank my supervisor Sergey Neshveyev for his help and precious advice throughout this work. I am grateful to Teodor Banica for useful suggestions. Thanks also to Marco Matassa for fruitful discussions.

\section{Singly Generated CAtegories of Hilbert spaces}

Our goal is to prove the following version of Woronowicz Tannaka-Krein duality.

1991 Mathematics Subject Classification. Operator Algebras, Quantum Algebra, Category Theory.

Supported by the European Research Council under the European Union's Seventh Framework Programme (FP/20072013)/ ERC Grant Agreement no. 307663 (PI: S. Neshveyev). 
Theorem 1.1. Let $H$ be a finite dimensional Hilbert space. Suppose we are given a collection $\mathcal{C}$ of spaces $\mathcal{C}(k, l)$ of operators $H^{\otimes k} \rightarrow H^{\otimes l}$ for all $k, l \geq 0$ satisfying the following properties:

(1) if $T, S \in \mathcal{C}$, then $T \otimes S \in \mathcal{C}$;

(2) if $T, S \in \mathcal{C}$ are composable, then $T S \in \mathcal{C}$;

(3) $T \in \mathcal{C}$ implies $T^{*} \in \mathcal{C}$;

(4) $\mathcal{C}(k, k)$ contains the identity operator for all $k \geq 0$;

(5) $\mathcal{C}(0,2)$ contains an operator $R$ such that $\left(R^{*} \otimes \iota\right)(\iota \otimes R)= \pm \iota$ on $H$.

Then there exists a unique up to isomomorphism compact quantum group $G$ with a self-conjugate fundamental representation $U$ on $H$ such that $\operatorname{Hom}_{G}\left(H^{\otimes k}, H^{\otimes l}\right)=\mathcal{C}(k, l)$ for all $k, l \geq 0$.

In the last section we will discuss how the general form of Woronowicz Tannaka-Krein duality can be easily deduced from this.

Denote by $\mathcal{A}$ the tensor algebra of the space of linear functionals on $B(H)$, i.e.,

$$
\mathcal{A}:=T\left(B(H)^{*}\right)=\bigoplus_{k=0}^{\infty} B\left(H^{\otimes k}\right)^{*} .
$$

Let $U \in B(H) \otimes B(H)^{*} \subset B(H) \otimes \mathcal{A}$ be the "fundamental matrix" of $\mathcal{A}$, so $U$ is characterized by the property

$$
(\iota \otimes T)(U)=T \quad \forall T \in B(H) .
$$

In other words,

$$
U:=\sum_{i, j} e_{i j} \otimes u_{i j}
$$

where the $e_{i j}$ 's are matrix units in $B(H)$ and $\left\{u_{i j}\right\}_{i, j}$ is the dual basis of $B(H)^{*}$ such that $u_{i j}\left(e_{k l}\right)=$ $\delta_{i k} \delta_{j l}$. The tensor algebra $\mathcal{A}$ is a bialgebra with comultiplication $\Delta$ defined by duality from the multiplication on $B(H)$, so that

$$
\Delta\left(u_{i j}\right)=\sum_{k} u_{i k} \otimes u_{k j},
$$

or equivalently, using the leg-numbering notation, $(\iota \otimes \Delta)(U)=U_{12} U_{13}$.

Next, denote by $\mathcal{A}_{n} \subset \mathcal{A}$ the subspace given by

$$
\mathcal{A}_{n}:=\bigoplus_{k=0}^{n} B\left(H^{\otimes k}\right)^{*}=\left(\bigoplus_{k=0}^{n} B\left(H^{\otimes k}\right)\right)^{*},
$$

and denote by $\mathcal{B}_{n}$ the commutant

$$
\mathcal{B}_{n}:=\left(\bigoplus_{k, l=0}^{n} \mathcal{C}(k, l)\right)^{\prime} \subseteq \bigoplus_{k=0}^{n} B\left(H^{\otimes k}\right) \subset B\left(\bigoplus_{k=0}^{n} H^{\otimes k}\right) .
$$

Finally, let

$$
\mathcal{I}_{n}:=\left\{a \in \mathcal{A}_{n}:\left.a\right|_{\mathcal{B}_{n}}=0\right\},
$$

and denote by $\mathcal{I}$ the union $\mathcal{I}:=\bigcup_{n=0}^{\infty} \mathcal{I}_{n}$. Note that $\mathcal{I}_{n+1} \cap \mathcal{A}_{n}=\mathcal{I}_{n}$, so $\mathcal{I}$ is a subspace of $\mathcal{A}$. Indeed, let $C_{n}=\left(\oplus_{k, l=0}^{n} \mathcal{C}(k, l)\right)=\mathcal{B}_{n}^{\prime}$ and denote by $p_{n}$ the projection $\oplus_{k=0}^{n+1} H^{\otimes k} \rightarrow \oplus_{k=0}^{n} H^{\otimes k}$. By definition $p_{n} \in C_{n+1}$ and $p_{n} C_{n+1} p_{n}=C_{n}$. Hence $\mathcal{B}_{n+1} p_{n}=\mathcal{B}_{n}$, by the Bicommutant Theorem (see e.g. [6], Section 3.).

Lemma 1.2. $\mathcal{I}$ is a bi-ideal in the bialgebra $\mathcal{A}$.

Proof. We will first prove that $\mathcal{I}$ is an ideal. Assume $a \in \mathcal{I}_{n}$ and $b \in B\left(H^{\otimes m}\right)^{*}$; we have to check that $a \otimes b$ vanishes on $\left(\oplus_{k, l=m}^{n+m} \mathcal{C}(k, l)\right)^{\prime}$. Since $\left(\oplus_{k, l=m}^{n+m} \mathcal{C}(k, l)\right)^{\prime} \subseteq\left(\oplus_{k, l=0}^{n} \mathcal{C}(k, l)\right)^{\prime} \otimes\left(\oplus_{k, l=0}^{m} \mathcal{C}(k, l)\right)^{\prime}$, the statement simply follows from the assumption that a vanishes on $\left(\oplus_{k=0}^{n} \mathcal{C}(k, l)\right)^{\prime}$.

To prove that $\mathcal{I}$ is a coideal we have to show that $\Delta(\mathcal{I}) \subseteq \mathcal{I} \otimes \mathcal{A}+\mathcal{A} \otimes \mathcal{I}$. For this purpose we use an equivalent definition of $\mathcal{I}$, that is, we consider the space spanned by the slices

$$
(\omega \otimes \iota)\left((T \otimes 1) U^{\otimes k}-U^{\otimes l}(T \otimes 1)\right)
$$

for all $\omega \in B\left(H^{\otimes k}, H^{\otimes l}\right)^{*}, T \in \mathcal{C}(k, l)$ and $k, l \geq 0$. This space indeed coincides with $\mathcal{I}$, since using (1.1) we see that an operator $S \in \oplus_{k=0}^{n} B\left(H^{\otimes k}\right)$ vanishes on the elements (1.2) for all $k, l \leq n$ if and only it 
lies in $\mathcal{B}_{n}$. We choose an orthonormal basis of $H^{\otimes k},\left\{\xi_{j}\right\}_{j}$, and of $H^{\otimes l},\left\{\eta_{i}\right\}_{i}$, and assume $\omega$ is of the form $\omega_{i j}=\left\langle\cdot \xi_{j}, \eta_{i}\right\rangle$. We set $V:=U^{\otimes k}$ and $W:=U^{\otimes l}$. Then, using the leg-numbering notation,

$$
\Delta\left(\omega_{i j} \otimes \iota\right)((T \otimes 1) V-W(T \otimes 1))
$$

is equal to

$$
\left(\omega_{i j} \otimes \iota \otimes \iota\right)\left((T \otimes 1 \otimes 1) V_{12} V_{13}-W_{12} W_{13}(T \otimes 1 \otimes 1)\right) .
$$

The expression in the parentheses can be written as

$$
\left((T \otimes 1 \otimes 1) V_{12}-W_{12}(T \otimes 1 \otimes 1)\right) V_{13}+W_{12}\left((T \otimes 1 \otimes 1) V_{13}-W_{13}(T \otimes 1 \otimes 1)\right) .
$$

Now, if we just consider the first part of the sum

$$
\left(\omega_{i j} \otimes \iota \otimes \iota\right)\left(\left((T \otimes 1 \otimes 1) V_{12}-W_{12}(T \otimes 1 \otimes 1)\right) V_{13}\right),
$$

it can be expressed as

$$
\sum_{k}\left(\omega_{i k} \otimes \iota \otimes \iota\right)\left((T \otimes 1 \otimes 1) V_{12}-W_{12}(T \otimes 1 \otimes 1)\right)\left(\omega_{k j} \otimes \iota \otimes \iota\right)\left(V_{13}\right),
$$

which belongs to $\mathcal{I} \otimes \mathcal{A}$. Similarly the other part lies in $\mathcal{A} \otimes \mathcal{I}$, as wanted.

By the previous Lemma, $\mathcal{A} / \mathcal{I}$ is a bialgebra (in the notation of [3], the description of $\mathcal{A} / \mathcal{I}$ via slice maps corresponds to the coend of the forgetful functor, End ${ }^{\vee}$ ). What we wish to prove is that $\mathcal{A} / \mathcal{I} \cong \mathrm{C}[G]$, for a compact quantum group $G$, and to do so we need for $\mathcal{A} / \mathcal{I}$ to be a Hopf *-algebra and for $U$ to be unitary (see Theorem 1.6.7 of [4]), and this is not obvious written in this manner. In fact it is not even clear whether $\mathcal{A} / \mathcal{I}$ has a $*$-structure. We shall proceed with an intermediate step. The idea is the following: we will introduce another bi-ideal in $\mathcal{A}, \mathcal{I}_{F}$, and show that $\mathcal{A} / \mathcal{I}_{F} \cong \mathrm{C}\left[O_{F}^{+}\right]$, where $O_{F}^{+}$is the free orthogonal quantum group. Thus, $\mathcal{A} / \mathcal{I}_{F}$ will automatically inherit a Hopf $*$-algebra structure. Finally we will show that $\mathcal{I} / \mathcal{I}_{F}$ is a Hopf $*$-ideal in $\mathcal{A} / \mathcal{I}_{F}$ and then conclude that there exists a compact quantum group $G$ such that

again by Theorem 1.6 .7 of [4].

$$
\mathcal{A} / \mathcal{I} \cong \mathcal{A} / \mathcal{I}_{F} / \mathcal{I} / \mathcal{I}_{F} \cong \mathrm{C}[G]
$$

\section{Representation CATEgory of a FREe orthogonal QuANTUM GRoup}

Following the strategy described above, we now consider the case when $\mathcal{C}$ is the smallest collection of spaces as in Theorem 1.1 containing a fixed operator $R: \mathrm{C} \rightarrow H^{\otimes 2}$ such that $\left(R^{*} \otimes \iota\right)(\iota \otimes R)= \pm \iota$. It is known, and not difficult to see, that if we fix an orthonormal basis $e_{1}, \ldots, e_{n}$ in $H$, then $R$ has the form $(\iota \otimes F) r$, where $r: \mathrm{C} \rightarrow H \otimes H$ is given by $r(1)=\sum_{i} e_{i} \otimes e_{i}$ and $F \in G L_{n}(\mathrm{C})$ is such that $F \bar{F}= \pm 1$, where $\bar{F}$ is the matrix obtained from $F$ by taking the complex conjugate of every entry. We will use the subindex $F$ for the constructions of the previous section related to this smallest collection, so we write $\mathcal{C}_{F}, \mathcal{B}_{F, n}, \mathcal{I}_{F, n}$, etc.

Consider the universal unital algebra $\mathrm{C}\left[O_{F}^{+}\right]$generated by entries of a unitary matrix $U=\left(u_{i j}\right)_{i, j}$ satisfying the relations

$$
U F^{t} U^{t}\left(F^{-1}\right)^{t}=1, \quad F^{t} U^{t}\left(F^{-1}\right)^{t} U=1 .
$$

for an invertible $n$ by $n$ matrix $F$. It is again known and easy to see that this is a Hopf $*$-algebra with comultiplication $\Delta\left(u_{i j}\right)=\sum_{k} u_{i k} \otimes u_{k j}$ and involution given by $U^{*}=F^{t} U^{t}\left(F^{-1}\right)^{t}$. The compact quantum group $O_{F}^{+}$thus defined is known in literature as the free orthogonal quantum group [7],[1], but we do not need to know any properties of this quantum group apart from the fact that it is well-defined. The following Lemma is a simple consequence of our definitions.

Lemma 2.1. We have $\mathcal{A} / \mathcal{I}_{F} \cong \mathrm{C}\left[O_{F}^{+}\right]$.

Proof. By definition, the bialgebra $\mathrm{C}\left[O_{F}^{+}\right]$can be written as $\mathcal{A} / \mathcal{L}$ where $\mathcal{L}$ is the ideal generated by the elements

$$
\left(F^{t}-\left(U F^{t} U^{t}\right)\right)_{i j}, \quad\left(\left(F^{-1}\right)^{t}-U^{t}\left(F^{-1}\right)^{t} U\right)_{i j} \quad \forall i, j
$$

with $U \in B(H) \otimes \mathcal{A}$ being the fundamental matrix of $\mathcal{A}$. In order to prove the Lemma we need to show that $\mathcal{I}_{F}=\mathcal{L}$. To show that $\mathrm{\textrm {L }} \subseteq \mathcal{I}_{F}$, consider the linear functionals $\omega_{1, i j}:=\left\langle\cdot 1, e_{i} \otimes e_{j}\right\rangle \in B\left(\mathrm{C}, H^{\otimes 2}\right)^{*}$ and $\omega_{i j, 1}:=\left\langle\cdot e_{i} \otimes e_{j}, 1\right\rangle \in B\left(H^{\otimes 2}, \mathrm{C}\right)^{*}$. Then

$$
\left(F^{t}-\left(U F^{t} U^{t}\right)\right)_{i j}=\left(\omega_{1, i j} \otimes \iota\right)\left((R \otimes \iota)-U^{\otimes 2}(R \otimes \iota)\right)
$$


and

$$
\left(\left(F^{-1}\right)^{t}-U^{t}\left(F^{-1}\right)^{t} U\right)_{i j}= \pm\left(\omega_{i j, 1} \otimes \iota\right)\left(\left(R^{*} \otimes \iota\right)-\left(R^{*} \otimes \iota\right) U^{\otimes 2}\right),
$$

where we recall that $R=(\iota \otimes F) r$ and in the second equality we use that $F^{*}= \pm\left(F^{-1}\right)^{t}$. Hence $\mathcal{L} \subseteq \mathcal{I}_{F}$.

Conversely, let us show that $\mathcal{I}_{F} \subseteq \mathcal{L}$. As follows from the above identities, $R$ and $R^{*}$ are morphisms in the category $\operatorname{Rep} O_{F}^{+}$. It follows that any operator in $\mathcal{C}_{F}$ is a morphism in $\operatorname{Rep} O_{F}^{+}$. But this implies $\mathcal{I}_{F} \subseteq \mathcal{L}$ because any relation defined by elements of $\mathcal{C}_{F}$ has to be satisfied in $\mathrm{C}\left[O_{F}^{+}\right]$.

\section{Proof of the Theorem}

We now turn to a general $\mathcal{C}$ as in Theorem 1.1. Let $R \in \mathcal{C}(0,2)$ be an operator such that $\left(R^{*} \otimes \iota\right)(\iota \otimes$ $R)= \pm \iota$. As in the previous section, we fix an orthonormal basis $e_{1}, \ldots, e_{n}$ in $H$ and write $R$ as $(\iota \otimes F) r$. Denote by $\mathcal{J}$ the bi-ideal $\mathcal{I} / \mathcal{I}_{F}$ in $\mathrm{C}\left[O_{F}^{+}\right]=\mathcal{A} / \mathcal{I}_{F}$. Note that $\mathcal{J}$ can still be described as the space spanned by the elements $(\omega \otimes \iota)\left((T \otimes 1) U^{\otimes k}-U^{\otimes l}(T \otimes 1)\right)$, for $T \in \mathcal{C}(k, l)$ and $\omega \in B\left(H^{\otimes k}, H^{\otimes l}\right)^{*}$, where we slightly abuse the notation and denote by the same symbol $U$ the fundamental matrix of $\mathcal{A}$ and its image in $\mathrm{C}\left[\mathrm{O}_{F}^{+}\right]$.

Lemma 3.1. $\mathcal{J}$ is a Hopf $*$-ideal in $\mathrm{C}\left[\mathrm{O}_{F}^{+}\right]$.

Proof. We denote by $S$ the antipode of $\mathrm{C}\left[O_{F}^{+}\right]$. Since $\mathcal{J}$ is a bi-ideal, we only need to check that $\mathcal{J}$ is closed under taking the adjoints and is invariant under $S$. Let $a^{*}=(\omega \otimes \iota)\left((T \otimes 1) U^{\otimes k}-U^{\otimes l}(T \otimes 1)\right)^{*}$.

We have to show that it lies in $\mathcal{J}$ for any $k, l \geq 0$. We first note that $\mathcal{C}(1,1)$ is closed under the operation $\vee$ defined by

$$
(\iota \otimes T) R=\left(T^{\vee} \otimes \iota\right) R, \text { since } T^{\vee}= \pm\left(\iota \otimes R^{*}\right)(\iota \otimes T \otimes \iota)(R \otimes \iota) .
$$

As $(\iota \otimes T) r=\left(T^{t} \otimes \iota\right) r$, we have $T^{\vee}=\left(F^{-1} T F\right)^{t}$, and the inverse operation, still preserving $\mathcal{C}(1,1)$, is $T \mapsto F T^{t} F^{-1}$. We recall from the previous section that we also have $U^{*}=F^{t} U^{t}\left(F^{-1}\right)^{t}$. Analogous formulas hold for $T \in \mathcal{C}(k, l)$. In fact, if we denote by $F_{k}=F^{\otimes k}$ and $U_{k}=U^{\otimes k}$,

then $T^{\vee}=\left(F_{l}^{-1} T F_{k}\right)^{t} \in \mathcal{C}(l, k)$ and $U_{k}^{*}=F_{k}^{t} U_{k}^{t}\left(F_{k}^{-1}\right)^{t}$. Therefore, choosing an orthonormal basis of $H^{\otimes k},\left\{\xi_{j}\right\}_{j}$, and of $H^{\otimes l},\left\{\eta_{i}\right\}_{i}$, and assuming $\omega$ is of the form $\omega_{i j}=\left\langle\cdot \xi_{j}, \eta_{i}\right\rangle$, we have

$$
\begin{aligned}
a^{*}= & \left(\omega_{i j} \otimes \iota\right)\left((T \otimes 1) U_{k}-U_{l}(T \otimes 1)\right)^{*}= \\
& \left(\omega_{j i} \otimes \iota\right)\left(U_{k}^{*}\left(T^{*} \otimes 1\right)-\left(T^{*} \otimes 1\right) U_{l}^{*}\right)= \\
& \left(\omega_{j i} \otimes \iota\right)\left(\left(\left(\left(T^{*}\right)^{t} \otimes 1\right)\left(U_{k}^{*}\right)^{t}-\left(U_{l}^{*}\right)^{t}\left(\left(T^{*}\right)^{t} \otimes 1\right)\right)^{t}\right)= \\
& \left(\omega_{i j} \otimes \iota\right)\left(\left(\left(T^{*}\right)^{t} \otimes 1\right)\left(U_{k}^{*}\right)^{t}-\left(U_{l}^{*}\right)^{t}\left(\left(T^{*}\right)^{t} \otimes 1\right)\right)= \\
& \left(\omega_{i j} \otimes \iota\right)\left(\left(\left(T^{*}\right)^{t} \otimes 1\right)\left(F_{k}^{-1} U_{k} F_{k}\right)-\left(F_{l}^{-1} U_{l} F_{l}\right)\left(\left(T^{*}\right)^{t} \otimes 1\right)\right)= \\
& \left(\omega_{i j} \otimes \iota\right)\left(\left(F_{l}^{-1} \otimes 1\right)\left(\left(F_{l}\left(T^{*}\right)^{t} F_{k}^{-1} \otimes 1\right) U_{k}-U_{l}\left(F_{l}\left(T^{*}\right)^{t} F_{k}^{-1} \otimes 1\right)\right)\left(F_{k} \otimes 1\right)\right)= \\
& \sum_{m, n}\left(F_{l}^{-1}\right)_{i m}\left(F_{k}\right)_{n j}\left(\omega_{m n} \otimes \iota\right)\left((\tilde{T} \otimes \iota) U_{k}-U_{l}(\tilde{T} \otimes \iota)\right),
\end{aligned}
$$

where $\tilde{T}=F_{l}\left(T^{*}\right)^{t} F_{k}^{-1} \in \mathcal{C}(k, l)$, since $T \mapsto F_{l} T^{t} F_{k}^{-1}$ is a map from $\mathcal{C}(l, k)$ to $\mathcal{C}(k, l)$, being the inverse operation of $\vee$. Hence, $a^{*} \in \mathcal{J}$.

The invariance of $\mathcal{J}$ under the antipode immediately follows from its invariance under involution. If $a=\left(\omega_{i j} \otimes \iota\right)\left((T \otimes 1) U_{k}-U_{l}(T \otimes 1)\right)$ then

$$
\begin{aligned}
S(a) & =\left(\omega_{i j} \otimes \iota\right)\left((T \otimes 1)(\iota \otimes S)\left(U_{k}\right)-(\iota \otimes S)\left(U_{l}\right)(T \otimes 1)\right) \\
& =\left(\omega_{i j} \otimes \iota\right)\left((T \otimes 1) U_{k}^{*}-U_{l}^{*}(T \otimes 1)\right) \\
& =\left(\omega_{j i} \otimes \iota\right)\left(U_{k}\left(T^{*} \otimes 1\right)-\left(T^{*} \otimes 1\right) U_{l}\right)^{*} \in \mathcal{J} .
\end{aligned}
$$

Given the above Lemma, we conclude that there exists a compact quantum group $G$ such that $\mathcal{A} / \mathcal{I} \cong$ $\mathrm{C}[G]$. It remains to show that $\operatorname{Hom}_{G}\left(H^{\otimes k}, H^{\otimes l}\right)=\mathcal{C}(k, l)$, and this is done in the following.

Lemma 3.2. We have $\operatorname{Hom}_{G}\left(H^{\otimes k}, H^{\otimes l}\right)=\mathcal{C}(k, l)$ for all $k, l \geq 0$. 
Proof. We have to show that for every $n$,

$$
\bigoplus_{k, l=0}^{n} \mathcal{C}(k, l)=\operatorname{End}_{G}\left(\bigoplus_{k=0}^{n} H^{\otimes k}\right) .
$$

As both sides are (finite dimensional) von Neumann algebras, the equality is equivalent to the equality of their commutants:

$$
\mathcal{B}_{n}=\operatorname{End}_{G}\left(\bigoplus_{k=0}^{n} H^{\otimes k}\right)^{\prime} .
$$

Recall now that for any finite dimensional representation $V \in B\left(H_{V}\right) \otimes \mathrm{C}[G]$ of a compact quantum group $G$ we have a representation $\pi_{V}$ of the algebra $\mathrm{C}[G]^{*}$ on $H_{V}$ defined by $\pi_{V}(\phi)=(\iota \otimes \phi)(V)$, and then $\pi_{V}\left(\mathrm{C}[G]^{*}\right)=\operatorname{End}_{G}\left(H_{V}\right)^{\prime}$. Therefore we have to show that $\pi_{\oplus_{k=0}^{n} U \otimes k}\left(\mathrm{C}[G]^{*}\right)=\mathcal{B}_{n}$. But this immediately follows from the previous Lemma, as $\mathrm{C}[G]=\mathcal{A} / \mathcal{I} \supset \mathcal{A}_{n} / \mathcal{I}_{n}=\mathcal{B}_{n}^{*}$.

To finish the proof of Theorem 1.1 we have to show that the compact quantum group $G$ is unique up to isomorphism. Let $G^{\prime}$ be another compact quantum group satisfying the assumptions of Theorem 1.1, that is, having a fundamental representation $V=\left(v_{i j}\right)_{i j}$ on $H$ such that $\operatorname{Hom}_{G^{\prime}}\left(H^{\otimes k}, H^{\otimes l}\right)=\mathcal{C}(k, l)$. We can identify $\mathrm{C}\left[G^{\prime}\right]$ with $\mathcal{A} / \mathcal{I}^{\prime}$, for a bi-ideal $\mathcal{I}^{\prime} \subset \mathcal{A}$. The only thing to check is that the bi-ideal $\mathcal{I}^{\prime}$ is completely determined by the operator spaces $\mathcal{C}(k, l)$. Since $\mathcal{C}(k, l)$ and $\operatorname{Hom}_{G^{\prime}}\left(H^{\otimes k}, H^{\otimes l}\right)$ coincide, from the proof of Lemma 3.2 we see that this implies that $\mathcal{A}_{n} / \mathcal{I}_{n}^{\prime}=\mathcal{B}_{n}^{*}$, where $\mathcal{I}_{n}^{\prime}=I^{\prime} \cap \mathcal{A}_{n}$ and $\mathcal{I}^{\prime}=\bigcup_{n \geq 0} \mathcal{I}_{n}^{\prime}$. Hence, the spaces $\mathcal{I}_{n}^{\prime}$ are completely determined by the spaces $\mathcal{C}(k, l)$. Thus Theorem 1.1 is proved.

\section{General version of the Tannaka-Krein duality}

In this section we want to explain, without too many details, how using Theorem 1.1 one can recover the following result.

Theorem 4.1 (Woronowicz Tannaka-Krein duality). Let $\mathcal{C}$ be an essentially small $C^{*}$-tensor category with conjugates, $\tau: \mathcal{C} \rightarrow$ Hilb $_{\mathrm{f}}$ be a unitary fiber functor. Then there exists a compact quantum group $G$ and a unitary monoidal equivalence $\theta: \mathcal{C} \rightarrow \operatorname{Rep} G$ such that $\tau$ is naturally unitarily monoidally isomorphic to the composition of the canonical fiber functor $\pi: \operatorname{Rep} G \rightarrow \operatorname{Hilb}_{\mathrm{f}}$ with $\theta$. Furthermore, the Hopf *-algebra $(\mathrm{C}[G], \Delta)$ for such a $G$ is uniquely determined up to isomorphism.

We remark that for $\mathrm{C}^{*}$-tensor categories we follow the conventions of [4], in particular, we assume that they are closed under finite direct sums and subobjects.

We concentrate only on the existence of $G$. We may assume that $\mathcal{C}$ is a subcategory of Hilb and $\tau$ is the embedding functor. If there exist an object $H$ in $\mathcal{C}$ such that any other object is isomorphic to a subobject of $H^{\otimes n}$ for some $n \geq 0$, and a morphism $R: C \rightarrow H \otimes H$ such that $\left(R^{*} \otimes \iota\right)(\iota \otimes R)= \pm \iota$, then the result follows from Theorem 1.1. For general $\mathcal{C}$ let us distinguish between three cases:

(i) $\mathcal{C}$ is generated, as a $\mathrm{C}^{*}$-tensor category with conjugates, by one object;

(ii) $\mathcal{C}$ is generated by a finite number of objects;

(iii) $\mathcal{C}$ is infinitely generated.

(i) Assume $\mathcal{C}$ is generated by one object $K$, so every object of $\mathcal{C}$ is isomorphic to a subobject of a tensor product of copies of $K$ and an object $\bar{K}$ conjugate to $K$. Let $\left(R^{\prime}, \bar{R}^{\prime}\right)$ be a solution of the conjugate equations for $K$ and $\bar{K}$. Then letting $H=K \oplus \bar{K}$ and $R=R^{\prime} \oplus \bar{R}^{\prime}$, considered as a morphism $\mathrm{C} \rightarrow H \otimes H$, we have $\left(R^{*} \otimes \iota\right)(\iota \otimes R)=\iota$, so we are back to the case covered by Theorem 1.1.

(ii) The case when $\mathcal{C}$ is generated by a finite number of objects $H_{1}, \ldots, H_{n}$ is not much different from (i), as then $\mathcal{C}$ is generated by $H_{1} \oplus \cdots \oplus H_{n}$.

(iii) For general $\mathcal{C}$, choose a generating set $\mathcal{F}$ in $\mathcal{C}$ and let $\mathcal{E}$ be the family of finite subsets of $\mathcal{F}$ ordered by inclusion. For each $E \in \mathcal{E}$ let $\mathcal{C}_{E}$ be the full rigid $\mathrm{C}^{*}$-tensor subcategory of $\mathcal{C}$ generated by the finite set of objects in $E$. By the previous case, for each subcategory $\mathcal{C}_{E}$ we get a compact quantum group $G_{E}$ with representation category $\mathcal{C}_{E}$. Moreover, if $E \subset E^{\prime}$, then, since $\mathcal{C}_{E} \subset \mathcal{C}_{E^{\prime}}$, by the uniqueness part of Theorem 1.1, the quantum group $G_{E}$ is a quotient of $G_{E^{\prime}}$, that is, we have an embedding $\mathrm{C}\left[G_{E}\right] \hookrightarrow \mathrm{C}\left[G_{E^{\prime}}\right]$ of Hopf $*$-algebras. Then $\mathrm{C}[G]$ is defined as the inductive limit of the Hopf *-algebras $\mathrm{C}\left[G_{E}\right]$.

In the following example we can see how to recover the free unitary quantum group following the procedure explained in point $(i)$ of the above. 
Example 4.2 (Free Unitary quantum group). We denote by $\mathrm{C}\left[U_{Q}^{+}\right]$the universal unital *-algebra generated by the entries of matrices $V=\left(v_{i j}\right)_{i, j}$ and $\bar{V}=\left(\bar{v}_{i j}\right)_{i, j}$ such that $V$ and $\bar{V}$ are unitary with involution defined by $V^{*}=Q^{t} \bar{V}^{t}\left(Q^{-1}\right)^{t}$ and $\bar{V}^{*}=\left(Q^{-1}\right)^{*} V^{t} Q^{*}$, for an invertible $n$ by $n$ matrix $Q$. The algebra $\mathrm{C}\left[U_{Q}^{+}\right]$is a Hopf *-algebra with comultiplication $\Delta\left(v_{i j}\right)=\sum_{k} v_{i k} \otimes v_{k j}$ and $U_{Q}^{+}$is known in literature as the free unitary quantum group.

We wish to prove the equivalence between the representation category of the free unitary quantum group and a concrete $C^{*}$-tensor category having certain properties. More specifically, consider the Hilbert space $K=\mathrm{C}^{n}$ and its complex conjugate $\bar{K}$. Let $\mathcal{C}_{Q}$ be the smallest collection of operators between tensor powers of $H:=K \oplus \bar{K}$, as in Theorem 1.1, containing the operator $R: C \rightarrow H^{\otimes 2}$ such that $\left(R^{*} \otimes \iota\right)(\iota \otimes R)=\iota$, and the projection $p: K \oplus \bar{K} \rightarrow K$. The operator $R$ is equal to $(\iota \otimes F) r$ for $F \in G L_{2}\left(M_{n}(\mathrm{C})\right)$ with entries $F_{11}=F_{22}=0, F_{12}=\bar{Q}^{-1}$ and $F_{21}=Q$, where $\bar{Q}$ is the matrix whose coefficients are the complex conjugates of the entries of $Q$.

We claim that $\operatorname{Hom}_{U_{Q}^{+}}\left(H^{\otimes k}, H^{\otimes l}\right)=\mathcal{C}_{Q}(k, l)$ for all $k, l \geq 0$. We show that $\mathrm{C}\left[U_{Q}^{+}\right] \cong \mathcal{A} / \mathcal{I}$, where $\mathcal{I}$ is the ideal generated by slice maps $(\omega \otimes \iota)\left((T \otimes 1) U^{\otimes k}-U^{\otimes l}(T \otimes 1)\right)$, for $T \in \mathcal{C}_{Q}(k, l)$ and $\omega \in$ $B\left(H^{\otimes k}, H^{\otimes l}\right)^{*}$. The claim will then follow from Theorem 1.1. By definition, $\mathrm{C}\left[U_{Q}^{+}\right]$can be written as $\mathcal{A} / \mathcal{L}$ where $\mathcal{L}$ is the ideal generated by the relations

$$
U F^{t} U^{t}\left(F^{-1}\right)^{t}=1, \quad F^{t} U^{t}\left(F^{-1}\right)^{t} U=1, \quad U_{12}=0, \quad U_{21}=0 .
$$

We already know that the ideal $\mathcal{I}$ contains slices of the first two relations, since we showed in Lemma 2.1 that they correspond to the slice maps

$$
\left(\omega_{1, i j} \otimes \iota\right)\left((R \otimes \iota)-U^{\otimes 2}(R \otimes \iota)\right)_{i, j}, \quad\left(\omega_{i j, 1} \otimes \iota\right)\left(\left(R^{*} \otimes \iota\right) U^{\otimes 2}-\left(R^{*} \otimes \iota\right)\right)_{i, j} .
$$

The other two relations correspond to $\left(\omega_{i j} \otimes \iota\right)((p \otimes \iota) U-U(p \otimes \iota))_{i, j}$. Hence $\mathcal{L} \subseteq \mathcal{I}$. The opposite inclusion follows analogously to the second part of the proof of Lemma 2.1.

\section{REFERENCES}

[1] T. Banica. Théorie des représentations du groupe quantique compact libre $O(n)$, C. R. Acad. Sci., 322, Série I,241-244, 1996.

[2] T. Banica and R. Speicher. Liberation of Orthogonal Lie groups. Advances in Mathematics, 222: 1461-1501, 2009.

[3] A. Joyal, R. Street. An introduction to Tannaka duality and quantum groups. Category theory (Como, 1990), 413-492, Lecture Notes in Math., 1488, Springer, Berlin, 1991.

[4] S. Neshveyev and L. Tuset. Compact quantum groups and their representation categories, volume 20 of Cours Spécialisés. Societe Mathématique de France, Paris, 2013.

[5] P. Schauenburg. Tannaka duality for Arbitrary Hopf Algebras. Algebra Berichte 66, Fischer, Munich, 1991.

[6] S. Strătilă, L. Zsidó Lectures on von Neumann Algebras, Editura Academiei/Abacus Press, Tunbridge Wells , 1979.

[7] A. Van Daele and S. Wang. Universal Quantum Groups. Int. J. Math., 7(2): 255-263, 1996.

[8] S. L. Woronowicz. Tannaka-Krein duality for compact matrix pseudogroups. Twisted SU(N) groups. Invent. Math., 93(1): 35-76, 1988.

Department of Mathematics, University of Oslo, P.O. Box 1053 Blindern, NO-0316 Oslo, Norway E-mail address: saramal@math.uio.no 